\title{
New Reactions Important for Analytical Chemistry
}

DOI: $10.1134 / \mathrm{S} 1061934810060018$

It seems that all this was in the past. The main chemical reactions that are of interest for analytical chemistry have been known for a long time. These are used even in relatively new (in any case, actively developed) analytical tools, test systems, optical sensors, etc. However, previously unknown reactions do appear sometimes; these are discovered occasionally, in some other study, or as a result a specific search; the possibilities of such searches increase because of the huge accumulated experience and the success of the theory. The search is quite often stimulated by an adequately formulated applied problem of current importance.

For example, the reaction of sulfonium borane with the cyanide ion was described; it provided a basis for the determination of fluorescence of cyanides in water, even in drinking water [1]. Borane (of complex structure) fluoresces and the presence of cyanide ions results in the quenching of fluorescence. The initial compound contains a coordinately unsaturated boron atom to which the cyanide ion present in water is attached.

Another example is provided by the reaction of the detection of low concentrations triacetone triperoxide (TATP), an explosive used by terrorists [2], which is hazardous because of its very easy synthesis. Upon UV irradiation, TATP releases hydrogen peroxide; the peroxide reacts with methyltrioxorhenium, which results in the oxidation of the reagent, whose molecule contains pyrene and a sulfoxide group. The sulfoxide group is oxidized to give fluorescent sulfone, and the detection of the explosive (down to $10^{-7} \mathrm{M}$ ) is based just on this fluorescence.

Currently, reactions for organic compounds, in particular for narcotics, and, of course, explosive substances, are of the greatest interest. Rapid chemical reactions of increased selectivity, which could be used in the field, with no risk of false signals from tobacco smoke, perfumes, etc., are of special importance. As for the reactions of traditional anions and especially metal cations, analysts have done much in this direction. This certainly does not mean that the search should be stopped, (see the example with the cyanide ion), but the urgency of this problem is appreciably not too high. We know that analysts from the former Soviet Union have made a huge contribution in this field of knowledge.

\section{REFERENCES}

1. Chem. Eng. News, 2009, vol. 87, no. 24, p. 27.

2. http://www.cen-online.org (September 29, 2008).

Yu. A. Zolotov 